\title{
Comparative Study of Disabled vs. Non-disabled Evaluators in User-Testing: Dyslexia and First Year Students Learning Computer Programming
}

\author{
Mark Dixon \\ University of Plymouth, School of Computing Communications and Electronics \\ Drake Circus, Plymouth, PL4 8AA, UK \\ mark.dixon@plymouth.ac.uk
}

\begin{abstract}
User-testing is a critical activity in software development. However, eliciting appropriate test-users can be difficult. Recent work showed that (during user-testing of educational software) dyslexic final-year students identified a larger number of subtle (yet significant) issues in more detail than other students. However, final year students were not the target users of the software (designed to teach fundamental programming concepts). This paper presents preliminary results of work replicating the previous study, but with participants from the target user group (first year students). The first year students identified fewer issues and gave less detail than the final year students. The dyslexic students identified more issues in greater detail than the other students. This highlights a distinction between the perceived target user group (first year students) and the actual target user group (students who don't understand programming concepts). Dyslexia may push people deeper into the actual target user group for educational software.
\end{abstract}

Keywords: User Testing, Software Evaluation, Educational Software, Dyslexia, Learning Computer Programming.

\section{Introduction}

\subsection{User Testing}

User testing is a critical activity in the software development lifecycle. All software systems rely upon it to ensure acceptability to users. There are many examples of significant usability issues being identified and hence resolved as a result of appropriate user testing (such as [1]).

However, gaining access to appropriate test-users can be difficult due to time and resource restraints, especially where the target users are highly skilled or qualified (such as physicians, solicitors, or lecturers). Many methods have been developed as substitutes for user testing (such as expert review, and cognitive walkthrough), but they can only reduce the amount of user testing required, they cannot replace it entirely [2]. The value of user testing is especially important in education, where poor software that confuses students can have a potentially life long impact. 


\subsection{Disabled Test-Users in Education}

Over the last five years software has been developed to animate the changes made by individual lines of program code to variables in memory. This code-memory diagram (CMD) animation software was designed to overcome the difficulties of teaching the fundamentals of computer programming, especially the limitations of oral narratives and white boards. Evaluations suggested that this software increases understanding [3] of students in the second, first, and foundation years of university (the foundation year is an access to Higher Education course intend for mature students who do not have the nominal entry qualifications). There are two parts to the software: a viewer (which is used to display animations to students during lectures and tutorials), and an editor (which is used to create and edit animations).

Recent work [4] showed that during user-testing of this software dyslexic students identified a larger number of subtle (yet significant) issues in more detail than other students, and also significant aspects of the learning and teaching process. It was suggested that these issues were relevant to the wider target user group. The study selected four final year students on the basis of availability and used a standard protocol to ensure that the same process (in particular the same set of questions) was applied consistently to all test-users. First year students were not used as it was thought they would be reluctant to provide constructive criticism of teaching (a phenomenon know as the 'A' level effect [5]).

\subsection{Target Users}

The use of final year students in user-testing for the CMD animation software is questionable, as the software was initially targeted for first year (introduction to programming) students. Also, it has never been used to teach final year students (only second, first, and foundation year). It is intended as an aide to learning structured and object-oriented programming concepts, which should be clearly understood by final year students. Hence, the issues identified by the final year students may be significantly different to those relevant to the target users (first year students).

\subsection{Comparison of Dyslexic and Non-dyslexic First Year Students as Evaluators}

This paper presents the preliminary results of work that replicates the previous (final year students) study, but with participants selected from the target user group (first year undergraduate degree students). A comparison is made of the feedback given by first-year students during software user testing, which followed the same protocol as the previous study with final year students. The issues identified by dyslexic and nondyslexic students are compared for both the first year students (in the present study) and the original four final stage students.

\section{Method}

A request for evaluators was sent (via email) to all students enrolled on a first year introduction to computer programming module delivered by the interviewer/researcher, 
where the CMD animation software (viewer) was used extensively. No financial (or other) incentive was offered for participation (as this may have biased the students in favour of the software). A total of four students were recruited. All were native English speakers, and had an established working relationship with the interviewer/researcher (via interaction during lectures and tutorials). Two of these students were reported as having dyslexia (referred to throughout this text as students $\mathrm{Sd} 3$, and $\mathrm{Sd} 4$ ).

The students were asked to use and evaluate two versions of the CMD Animation Viewer (Vi1 and Vi2) and two versions of the CMD Animation Editor software (Ed1 and Ed2). A single example animation was used for all pieces of software. This animation involved nine fundamental (year 1) computer programming concepts: variable declaration and assignment, array declaration and assignment, local variables, module level variables, a for loop, and a function declaration and call. All of these concepts were explicitly covered in the module attended by the students, which included the use of animation viewer (Vi2) embedded into PowerPoint slides.

The same protocol developed in the previous study was used, to ensure that the same process (in particular the same set of questions) was applied consistently to all participants, and leading questions were avoided. The evaluations were conducted independently one student at a time, outside normal teaching sessions. Participation was voluntary and started with the interviewer briefing the participant.

The students were asked some background questions regarding age, number of years programming experience, and whether they had any registered special needs. Students were invited to 'think-aloud' [1], and interrupt the process to comment on any aspects of the software's impact on their understanding of the computer programming concepts in the example animation, as well as aspects of the software that they felt were good and aspects that could be improved. The four software applications were then demonstrated, and in the case of the editors the student was invited to use the software. After each software application was demonstrated the student was asked to comment.

Audio recordings were made of each session, and transcribed. Recurrent themes were then identified, with particular attention to issues reported by the students regarding difficulties in computer programming learning and teaching, positive characteristics of the software, and criticisms/suggestions for improvement of the software. These themes/issues were emergent from the data (rather than being predetermined), but were subsequently cross referenced against the themes identified by the four final year students in the previous study (S1, S2, Sd1, Sd2).

\subsection{Animation Viewers}

Figure 1 shows version 1 of the CMD animation viewer software (Vi1), which was designed as a presentation aid to support narrative explanations of code execution delivered by lecturers to students. As each line is executed a tick appears to its left, and its impact on variables is reflected in the memory diagram (right). 


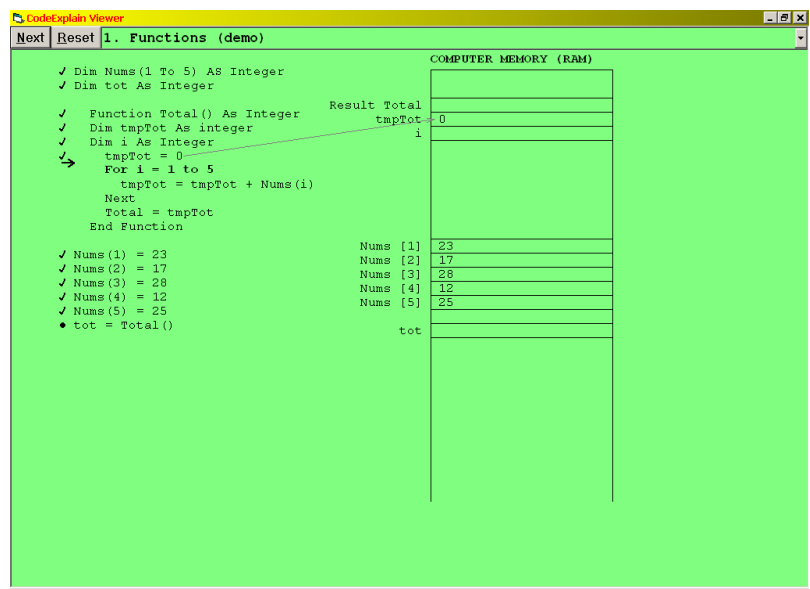

Fig. 1. CMD Animation Viewer software - version 1 (Vi1)

Figure 2 shows version 2 of the CMD animation software viewer (Vi2), which was familiar to the first years students (as it was used in their programming lectures).

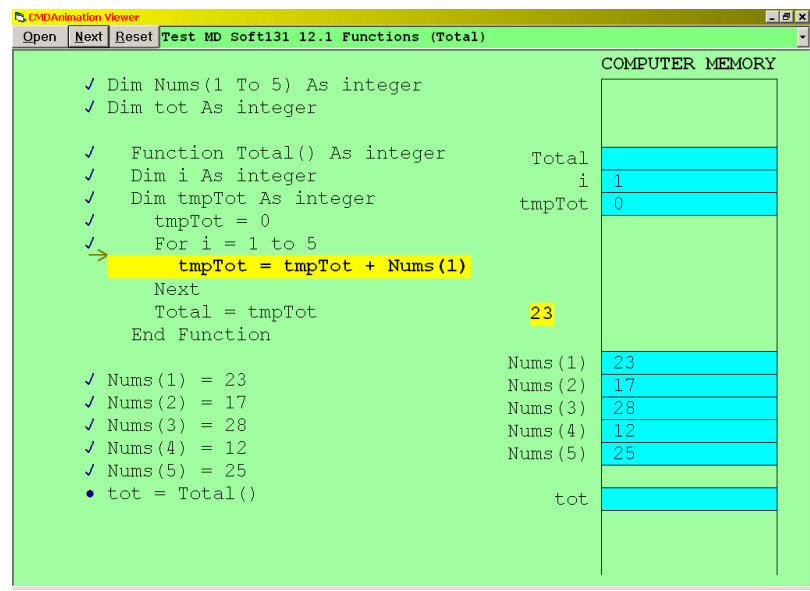

Fig. 2. CMD Animation Viewer software - version 2 (Vi2)

It incorporated additional functionality as a result of suggestions from users (both students and lecturers):

- Variable font size - allowed the lecturer to change the size of the font, in order to present the animations as large as possible, while still fitting on the screen.

- Transitional animation - animated the processes of:

- variable creation (where the variable name flew from the line of code which created it to the position in memory which it occupied), 
- variable assignment (where the value of the expression on the right-hand side of an assignment statement flew from the line of code to the variable on the memory diagram, mentioned in the left-hand side of the assignment statement), and

- variable removal (where as a variable went out of scope, it was removed from the memory diagram by flying from the diagram to the line that caused it to go out of scope).

- Current line highlighted background - The current line of program code was highlighted yellow.

- Variable name-value substitution animation - The process that replaces the names of variables in expressions with their current values was animated, so that the values moved from their position in the memory diagram to replace the names of their respective variables in the line of code which referred to it.

\subsection{Animation Editors}

Figure 3 shows version 1 of the CMD animation editor software (Ed1), which was designed to help lecturers create animations and modify animations to meet the specific needs of their student groups. It suffered from significant usability limitations, which made its use in routine teaching impractical (as creating and modifying animations was difficult and time consuming).

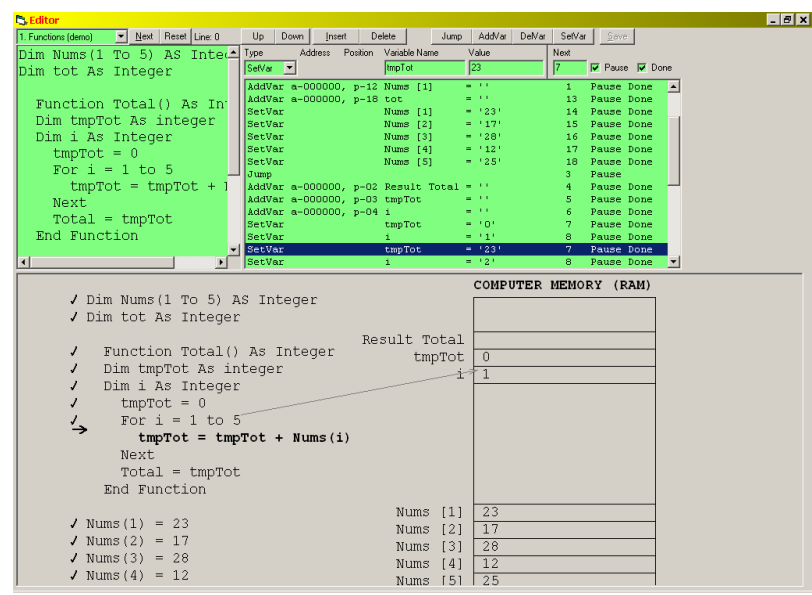

Fig. 3. CMD Animation Editor - version $1(\mathrm{Ed} 1)$

Figure 4 shows version 2 of the CMD animation editor software, which was developed as a result of limitations with the initial editor (Ed1). This employed a direct-manipulation [6] user-interface to increase usability, and hence make the process of creating animations faster and easier for lecturers. The example animation included all 6 frame types available in this version of the editor: create variable, set variable, remove variable, call, do, and substitute (not available in Vi1 or Ed1). 


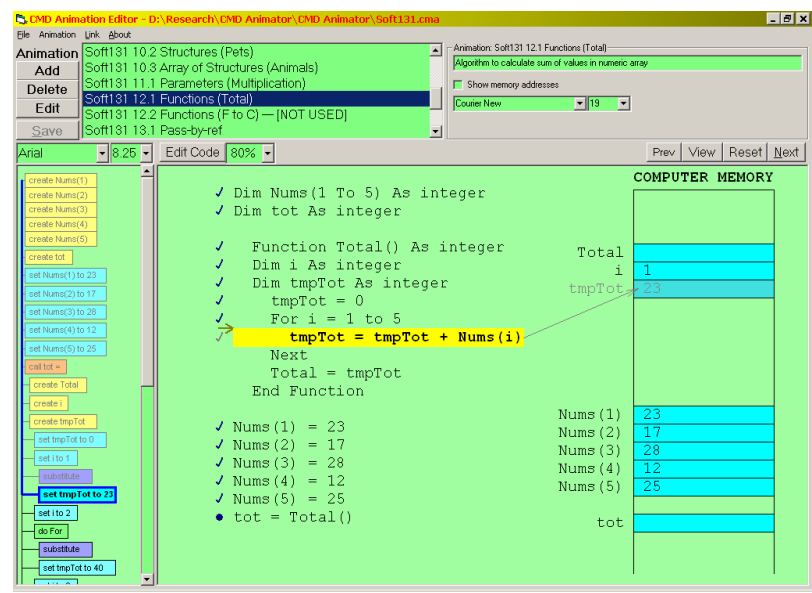

Fig. 4. CMD Animation Editor - version $2(\mathrm{Ed} 2)$

\section{Results}

One student (S4) was completely unfamiliar with programming (having come from an art and design background) and as a result had considerable contact with the lecturer. Two students ( $\mathrm{Sd} 3, \mathrm{Sd} 4)$ had a limited exposure to typing in scripts prior to their enrolment on the current degree course, but had not been taught computer programming formally. Student $(\mathrm{Sd} 4)$ had very little contact with the lecturer. One student (S3) had withdrawn from a computing degree 10 years earlier.

\subsection{Computer Programming Learning and Teaching Difficulties}

One of the students ( $\mathrm{Sd} 4)$ described a learning and teaching difficulty. Table 1 shows this along with the data from the previous study (with final year students).

Table 1. Summary of difficulties with the learning and teaching of computer programming, reported by the student evaluators

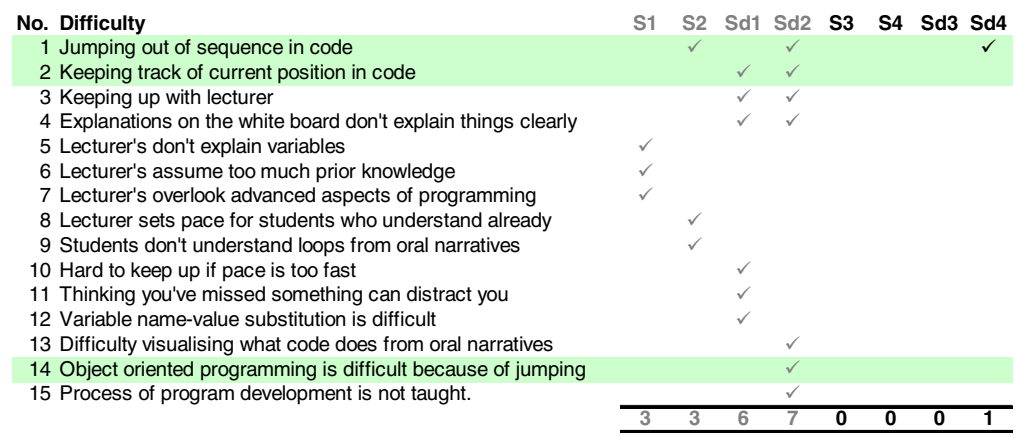


The only difficulty described, involved jumping out of sequence in code. Student $\mathrm{Sd} 4$ commented that it was 'quite logical to go through the code' line after line, but without the animation software 'jumps' between function call and definition would be difficult to follow [741]. The number in square brackets, indicates which paragraph in the transcript the quote is taken from (i.e. paragraph seven hundred and forty one).

\subsection{Software: Positive Characteristics}

The first year students described 13 positive characteristics of the four software applications, which were also described by the final year students. Table 2 shows which students reported each positive characteristic, and the total number of positive characteristics reported by each student, including the original final year students ( $\mathrm{S} 1$, $\mathrm{S} 2$, Sd1, and Sd2).

Table 2. Summary of positive characteristics of the four software applications (Vi1, Vi2, Ed1, and $\mathrm{Ed} 2$ ) reported by the student evaluators

No. Positive Characteristic
1 V1: Visual element
2 V1: Step-by-step explanation
3 V1: Ticks
4 V1: Contents of variables
5 V2: Yellow Highlight of Current Line
6 V2: Transitional Animation
7 V2: easier to see where you are
8 V2: easier to keep up/catch up
9 V2: Substitution
10 V2: Larger Font
11 V2: Actually doing
12 V2: Predict what happens next
13 V2: Arrows
14 V2: Memory Variable Background
15 V2: Previous perception of Substitution
16 E2: Drag and drop
17 E2: Font Size
18 E2: Help lecturer's understanding of students
19 E2: Colour coded
20 E2: Font used to shrink down, for large animations
21 E2: Immediate visual feedback
22 E2: Save button
23 E2: Slows delivery
Overall Total

No. Positive Characteristic

1 1 1 : Visual element

3 V1: Ticks

6 V2: Transitional Animation

7 V2: easier to see where you are

10 V2: Larger Font

16 E2: Drag and drop

19 E2: Colour coded

0 E2: Font used to shrink down, for large animations

22 E2: Save button

Overall Total
13 V2: Arrows

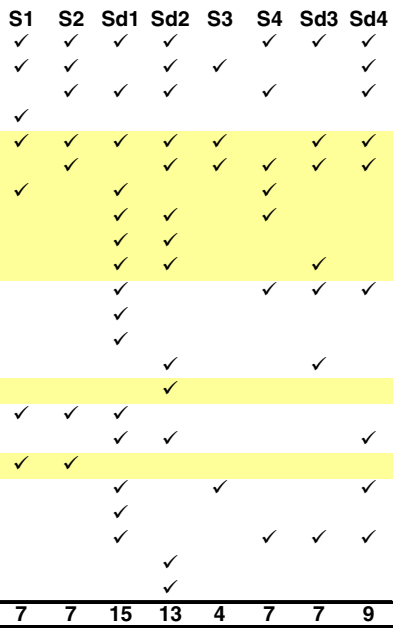

The following examples are representative of the type of comments made by the four first year students:

- Yellow Highlight of Current Line: Three of the first year students (S3, Sd3, and $\mathrm{Sd} 4$ ) commented on the yellow highlighting of the current line in version 2 of the viewer. In particular, student Sd4 indicated that it helped address the difficulty associated with the execution point jumping in function calls - 'the highlight took [animated] it up' to the function definition whereas the 'old one ... just went [jumped]' straight up to the definition [777].

- Transitional Animation: All four first year students commented on the transitional animation. Student S4 said 'I like the fact that ... the numbers physically move across the page' [286]. Student Sd4 commented that 'the [transitional] animation leads to different things ... you can see where you're going with each bit of code' [775]. 


\subsection{Software: Negative Characteristics and Suggestions for Improvement}

The students described 7 negative characteristics/suggestions for improvement of the four software applications. Three of these were not mentioned in the previous (final year student) study. Table 3 shows which students reported each characteristic/ suggestion, and the total number of characteristics/suggestions reported by each student. The students with dyslexia $(\mathrm{Sd} 3$, and $\mathrm{Sd} 4)$ described 2 and 6 characteristics respectively, and student (S3) described 1 characteristic.

Table 3. Summary of negative characteristics and suggestions for improvement of the four software applications (Vi1, Vi2, Ed1, and Ed2) reported by the student evaluators

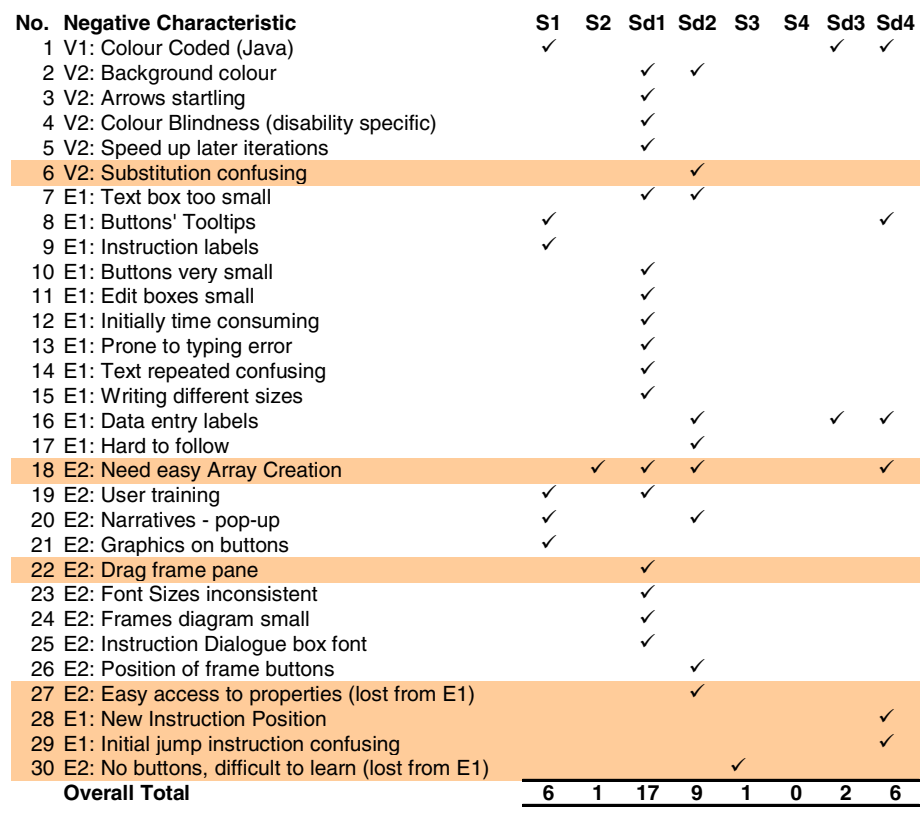

The following examples are representative of the comments made by the students:

- Easy array creation: While creating the frames for animating the declaration of an array, student $\mathrm{Sd} 4$ asked if there was 'a way of replicating your last moves' [973], and that if there were it would save 'having to go back and forth' between the code and the memory diagram [979].

\subsection{Subtle Significant Issues}

Table 4 shows a summary of which students reported issues regarded by the developer as both subtle (surprising to the developer, and not considered prior to feedback from others) and significant (relevant to the general student population and would directly influence future software design and/or teaching practice). 
Table 4. Summary of issues regarded by developer as both subtle and significant to the general student population, with indication of which students reported them

\begin{tabular}{|c|c|c|c|c|c|c|c|c|c|}
\hline & No. Positive Characteristic & s1 & S2 & Sd1 & Sd2 & S3 & S4 & Sd3 & Sd4 \\
\hline Difficulties & $\begin{array}{l}1 \text { Jumping out of sequence in code } \\
2 \text { Keeping track of current position in code } \\
14 \text { Object oriented programming is difficult because of jumping }\end{array}$ & & $\checkmark$ & $\checkmark$ & $\begin{array}{l}\checkmark \\
\checkmark \\
\checkmark\end{array}$ & & & & $\checkmark$ \\
\hline $\begin{array}{r}\text { Positive } \\
\text { Characteristics }\end{array}$ & $\begin{array}{l}5 \text { Vi2: Yellow Highlight of Current Line } \\
6 \text { Vi2: Transitional Animation } \\
7 \quad \text { Vi2: easier to see where } \\
8 \quad \text { Vi2: easier to keep up } \\
9 \text { Vi2: Substitution } \\
10 \text { Vi2: Larger Font } \\
15 \quad \text { Vi2: Changed perception of substitution } \\
18 \text { Ed2: Help lecturer's understanding of students }\end{array}$ & $\checkmark$ & $\checkmark$ & $\begin{array}{l} \\
\checkmark \\
\checkmark \\
\checkmark \\
\checkmark\end{array}$ & $\begin{array}{l}\checkmark \\
\checkmark \\
\checkmark \\
\checkmark \\
\checkmark \\
\checkmark\end{array}$ & $\begin{array}{l}\checkmark \\
\checkmark\end{array}$ & $\begin{array}{l}\checkmark \\
\checkmark \\
\checkmark\end{array}$ & $\checkmark$ & $\begin{array}{l}\checkmark \\
\checkmark\end{array}$ \\
\hline $\begin{array}{r}\text { Negative } \\
\text { Characteristics }\end{array}$ & $\begin{array}{l}6 \text { Vi2: Substitution confusing } \\
18 \text { Ed2: Easy Array Creation } \\
22 \text { Ed2: Drag frame pane } \\
27 \text { Ed2: Easy access to properties (lost from e1) } \\
28 \text { E1: New Instruction Position } \\
29 \text { E1: Initial jump instruction confusing } \\
30 \text { E2: No buttons, difficult to learn (lost from E1) }\end{array}$ & & $\checkmark$ & $\begin{array}{l}\checkmark \\
\checkmark\end{array}$ & $\begin{array}{l}\checkmark \\
\checkmark \\
\checkmark\end{array}$ & $\checkmark$ & & & $\begin{array}{l}\checkmark \\
\checkmark\end{array}$ \\
\hline Total & & 3 & 5 & 8 & 12 & 3 & 3 & 3 & 6 \\
\hline
\end{tabular}

\section{Conclusions}

\subsection{Comparison of Year Groups}

Overall the first year students identified fewer issues and described them in less detail than the final year students. This suggests that first year students may be reluctant to provide constructive criticism of teaching methods and tools, and that the original strategy of using final year rather than first year students was appropriate.

It is possible that the viewer software (V2) was so heavily embedded in the first year students' learning experience, that it had become tacit, which reduced their ability to criticise it (although they were unfamiliar with V1, E1, or E2). It may be that the use of the software prevented these students from experiencing many of the usual difficulties associated with learning computer programming (it worked as intended), and this made it difficult for them to articulate or be aware of these issues.

Also, final year students tend (one would hope) to be more confident, articulate, have stronger critical reasoning skills, and be more familiar with computing concepts.

\subsection{Comparison of Dyslexic vs. Non-dyslexic Groups}

Overall, the dyslexic students identified more issues in a greater amount of detail than the other students. However, the difference was far less distinct than with the final year students. Again, the use of the software in normal teaching may have reduced their exposure to difficulties, which they are usually more sensitive to than other students. Also, there was a marked difference between the two dyslexic students; student Sd3 behaved equivalently to the two non-dyslexic students, but student $\mathrm{Sd} 4$ showed a considerable difference (similar to the two dyslexic final year students). This is especially interesting as student Sd4 was the youngest evaluator, and had the least established working relationship with the lecturer (infrequent tutorial contact). 


\subsection{Perceived vs. Actual Target User Group}

This study has highlighted a distinction between the perceived target user group (first year students) and the actual target user group (students who don't understand programming concepts). Hence, actual target user groups for educational software may be more usefully defined in terms of their (lack of) understanding of certain concepts, rather than their age or organisational grouping (class, year group, degree programme). In practice this is very difficult to achieve, requiring extensive summative assessment. A final year student who has struggled and only just grasped certain concepts, may be a more effective evaluator of educational software than a first year who has been exposed to the concepts for several years.

The first year students all indicated that they found the animation software useful during the normal teaching sessions, and that they understood the fundamental programming concepts covered by the sample animation. A perception supported by their grades (61\% average for 4 students). This effectively pushes them outside the actual target user group (students who don't understand programming concepts).

It is widely accepted that the presence of dyslexia in a student who is learning computer programming makes the experience more difficult. Also, the symptoms of dyslexia are often described as being common problems for everyone, that are experienced to a much greater degree in some people (dyslexics).

A possible explanation for the results of the present work is that this additional difficulty makes the dyslexic person more aware of the learning and teaching process, especially the problems. They have to consciously consider issues that other students deal with instinctively, and these experiences therefore remain memorable for much longer. In effect, the dyslexia may push people deeper into the actual target user group (people who don't understand) for educational software.

Many avenues of further work remain unexplored. It would be interesting (but very difficult) to use the same protocol on students who fail or have withdrawn from the degree programme due to difficulties understanding computer programming. In theory they should be at the centre of the actual target user group and may provide the most insightful comments.

\section{References}

1. Nielsen, J.: Usability Engineering. Academic Press Inc, London (1993)

2. Dix, A., Finlay, J., Abowd, G., Beale, R.: Human-Computer Interaction, 3rd edn. Pearson Education Limited, London (2004)

3. Dixon, M.: Generic Code-Memory Diagram Animation Authoring Tool: Impact on Learning and Teaching Object-Oriented Programming. In: Proceedings of the 2nd International conference on Education and Technology, Calgary, Canada, 17-19 July, pp. 84-89 ACTA Press. (2006)

4. Dixon, M.: Code-Memory Diagram Animation Software for Teaching Computer Programming: contributions for and by dyslexic students. In: Accepted for 3rd International Conference on Universal Access in Human-Computer Interaction, Las Vegas, (July 22-27, 2005)

5. Bryson, J.: Breaking through the A Level Effect: a first-year tutorial in student selfreflection. Journal of Geography in Higher Education 21(2), 163-169 (1997)

6. Shneiderman, B.: Designing the user interface: Strategies for Effective Human-Computer Interaction, 3rd edn. Addison-Wesley Publishing Company Inc, Redwood City (1998) 